Design and Manufacture

An Integrated Approach 


\section{Other Macmillan titles of interest to engineers}

Acoustics for Engineers, John Turner and A. J. Pretlove

CAD/CAM: Features, Applications and Management, Peter F. Jones

Engineering Mathematics, Third Edition, Ken Stroud

Form, Structure and Mechanism, M. J. French

Further Engineering Mathematics, Second Edition, Ken Stroud

Introduction to Engineering Materials, Third Edition, Vernon John

Introduction to Internal Combustion Engines, Second Edition, Richard Stone

Motor Vehicle Fuel Economy, Richard Stone

Tribology: Principles and Design Applications, D. Arnell, P. Davies, J. Halling and T. Whomes

\section{Foundations of engineering}

Dynámics, G. E. Drabble

Electric Circuits, P. Silvester

Electromagnetism, R. G. Powell

Fluid Mechanics, Martin Widden

Structural Mechanics, J. A. Cain and R. Hulse

Thermodynamics, J. Simonson 


\title{
Design and Manufacture
}

An Integrated Approach

\author{
Rod Black \\ University of Portsmouth
}


(C) R. M. Black 1996

All rights reserved. No reproduction, copy or transmission of this publication may be made without written permission.

No paragraph of this publication may be reproduced, copied or transmitted save with written permission or in accordance with the provision of the Copyright, Designs and Patents Act 1988, or under the terms of any licence permitting limited copying issued by the Copyright Licensing Agency, 90 Tottenham Court Road, London W1P 9HE.

Any person who does any unauthorised act in relation to this publication may be liable to criminal prosecution and civil claims for damages.

First published 1996 by

MACMILLAN PRESS LTD

Houndmills, Basingstoke, Hampshire RG21 6XS

and London

Companies and representatives

throughout the world

ISBN 978-0-333-60915-6 ISBN 978-1-349-13429-8 (eBook)

DOI 10.1007/978-1-349-13429-8

A catalogue record for this book is available from the British Library.

$\begin{array}{llllllllll}10 & 9 & 8 & 7 & 6 & 5 & 4 & 3 & 2 & 1\end{array}$

$\begin{array}{llllllllll}05 & 04 & 03 & 02 & 01 & 00 & 99 & 98 & 97 & 96\end{array}$

Copy-edited and typeset by Povey-Edmondson

Okehampton and Rochdale, England 
To the next generation, particularly Ben, Doug and Alex 


\section{Contents}

Preface $\quad$ xv

Acknowledgements $\quad$ xvii

1 Concept to Solution - An Overview 1

1.1 Introduction 1

$\begin{array}{lll}1.2 & \text { New product introduction process } & 1\end{array}$

1.1.2 Background 1

1.2.2 Need 3

1.2.3 Engineering model 3

1.2.4 Design detail 3

$\begin{array}{lll}1.2 .5 & \text { Prototype } & 4\end{array}$

1.2.6 Field trials 4

1.2.7 Pre-production $\quad 4$

1.2.8 Integrated approach $\quad 6$

$\begin{array}{ll}1.2 .9 & \text { Control system }\end{array}$

$\begin{array}{lll}1.3 & \text { Summary } & 7\end{array}$

1.4 Questions $\quad 7$

\section{Part I The Basics of Design}

2 Records and Communication: The Engineering Drawing $\quad 11$

$2.1 \quad$ Introduction $\quad 11$

2.2 Engineering drawings 11

$\begin{array}{lll}2.2 .1 & \text { Equipment } & 12\end{array}$

2.2.2 Drawing layout $\quad 12$

$\begin{array}{lll}2.2 .3 & \text { Sketching } & 12\end{array}$

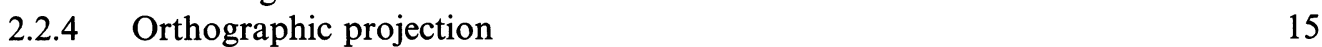

$\begin{array}{lll}2.2 .5 & \text { Sections } & 18\end{array}$

2.2.6 Auxiliary projection $\quad 19$

2.2.7 Types of drawings $\quad 21$

$\begin{array}{lll}2.2 .8 & \text { Dimensions } & 23\end{array}$

2.2.9 Abbreviations and standard components $\quad 25$

2.3 Maintaining records $\quad 26$

2.3.1 Drawing identification $\quad 26$

$\begin{array}{ll}\text { 2.3.2 The working file } & 26\end{array}$

$\begin{array}{lll}2.4 & \text { Summary } & 27\end{array}$

$\begin{array}{llr}2.5 & \text { Questions } & 28\end{array}$ 
3 The Importance of Detail: Tolerances $\quad 30$

$\begin{array}{llr}3.1 & \text { Tolerances } & 30\end{array}$

$\begin{array}{lll}3.1 .1 & \text { Introduction } & 30\end{array}$

$\begin{array}{lll}3.1 .2 & \text { Definition } & 30\end{array}$

3.1.3 Cost implications of tolerances $\quad 31$

$\begin{array}{lll}3.1 .4 & \text { Presentation } & 32\end{array}$

$\begin{array}{lll}3.1 .5 & \text { Functional dimensions } & 32\end{array}$

$\begin{array}{lll}3.1 .6 & \text { Choice of datum } & 33\end{array}$

$\begin{array}{lll}3.1 .7 & \text { Tolerance allocation } & 34\end{array}$

$\begin{array}{ll}3.1 .8 & \text { Allocation examples }\end{array}$

$\begin{array}{lll}3.2 & \text { Limits and fits } & 38\end{array}$

$\begin{array}{lll}3.2 .1 & \text { Introduction } & 38\end{array}$

$\begin{array}{lll}3.2 .2 & \text { BS4500 } & 38\end{array}$

$\begin{array}{lll}3.2 .3 & \text { Tolerance grade } & 38\end{array}$

$\begin{array}{lll}3.2 .4 & \text { Deviation } & 39\end{array}$

$\begin{array}{lll}3.2 .5 & \text { Syntax } & 39\end{array}$

$\begin{array}{lll}3.2 .6 & \text { Types of fit } & 40\end{array}$

3.2.7 Hole or shaft base? $\quad 40$

$3.3 \quad$ Surface finish $\quad 41$

3.3.1 Introduction $\quad 41$

$\begin{array}{lll}3.3 .2 & \text { Assessment } & 42\end{array}$

$\begin{array}{lll}\text { 3.3.3 Syntax } & 42\end{array}$

$\begin{array}{lll}3.3 .4 & \text { Cost } & 43\end{array}$

$3.4 \quad$ Geometric tolerances $\quad 44$

$\begin{array}{lll}3.4 .1 & \text { Introduction } & 44\end{array}$

$\begin{array}{lll}3.4 .2 & \text { Syntax } & 44\end{array}$

3.4.3 Geometric tolerancing examples $\quad 46$

3.4.4 When to use geometric tolerancing $\quad 49$

$\begin{array}{ll}3.5 & \text { Maximum material condition }\end{array}$

3.6 Examples $\quad 51$

3.7 Summary $\quad 53$

$\begin{array}{lll}3.8 & \text { Questions } & 53\end{array}$

4 Standard Components $\quad 54$

$4.1 \quad$ Introduction $\quad 54$

4.2 Types of standard $\quad 54$

4.3 Types of transmission $\quad 55$

4.3.1 Introduction $\quad 55$

4.3.2 Shafts, loads, keys and keyways $\quad 56$

$\begin{array}{lll}\text { 4.3.3 Belt transmissions } & 58\end{array}$

$\begin{array}{ll}\text { 4.3.4 Chains and timing belts } & 62\end{array}$

$\begin{array}{lll}\text { 4.3.5 Gears } & 62\end{array}$

$\begin{array}{lll}\text { 4.3.6 Hydraulic transmissions } & 68\end{array}$

$\begin{array}{ll}\text { 4.3.7 Electric transmissions } & 69\end{array}$

$\begin{array}{lll}4.4 & \text { General standard components } & 71\end{array}$

$\begin{array}{lll}4.4 .1 & \text { Couplings } & 71\end{array}$

$\begin{array}{lll}\text { 4.4.2 Clutches } & 73\end{array}$

$\begin{array}{lll}4.4 .3 & \text { Bearings } & 74\end{array}$ 
$\begin{array}{lll}4.4 .4 & \text { Seals } & 82\end{array}$

$\begin{array}{lll}4.5 & \text { Joining devices } & 84\end{array}$

$\begin{array}{lll}\text { 4.5.1 Threaded fasteners } & 84\end{array}$

4.5.2 Non-threaded fasteners

$\begin{array}{llr}4.6 & \text { Summary } & 89\end{array}$

$\begin{array}{llr}4.7 & \text { Questions } & 89\end{array}$

5 The Design Process $\quad 91$

$\begin{array}{lll}5.1 & \text { Introduction } & 91\end{array}$

5.2 The process $\quad 91$

5.2.1 Definition $\quad 92$

$\begin{array}{lll}5.3 & \text { Product design specification } & 92\end{array}$

5.4 Elements of the PDS $\quad 94$

$\begin{array}{ll}\text { 5.4.1 Origin of need } & 94\end{array}$

$\begin{array}{ll}\text { 5.4.2 Operation } & 94\end{array}$

$\begin{array}{ll}5.4 .3 \text { Life } & 96\end{array}$

5.4.4 Producer 98

$\begin{array}{ll}\text { 5.4.5 Society } & 99\end{array}$

$\begin{array}{lr}\text { 5.4.6 Customer } & 99\end{array}$

$\begin{array}{lll}5.5 & \text { Information sources } & 101\end{array}$

$\begin{array}{ll}\text { 5.5.1 Standards } & 101\end{array}$

5.5.2 Component manufacturers 101

$\begin{array}{ll}\text { 5.5.3 Competitors' products } & 101\end{array}$

$\begin{array}{ll}\text { 5.5.4 Library searches } & 101\end{array}$

$\begin{array}{ll}\text { 5.5.5 Patents } & 102\end{array}$

$\begin{array}{lll}5.6 & \text { Idea generation } & 103\end{array}$

5.6.1 Common obstacles 103

$\begin{array}{ll}\text { 5.6.2 Improving creativity } & 104\end{array}$

$\begin{array}{llr}5.7 & \text { Idea evaluation } & 108\end{array}$

5.7.1 Initial screening $\quad 108$

$\begin{array}{ll}\text { 5.7.2 Concept comparison } & 108\end{array}$

5.7.3 Criteria weighting 110

5.8 Summary 111

5.9 Questions 112

\section{Part II The Basics of Manufacture}

6 The Manufacturing Process $\quad 115$

$\begin{array}{ll}\text { 6.1 Introduction } & 115\end{array}$

6.2 Material selection $\quad 115$

$\begin{array}{lll}\text { 6.2.1 Importance of materials } & 115\end{array}$

6.2.2 Factors influencing selection $\quad 116$

$\begin{array}{lll}\text { 6.3 Manufacturing processes } & 117\end{array}$

$\begin{array}{lll}\text { 6.3.1 Shape modifying processes } & 117\end{array}$

$\begin{array}{ll}\text { 6.3.2 Property modifying processes } & 120\end{array}$

$\begin{array}{lll}\text { 6.3.3 Joining processes } & 121\end{array}$

$\begin{array}{lll}6.4 & \text { Manufacturing system } & 121\end{array}$

$\begin{array}{llr}6.5 & \text { Summary } & 121\end{array}$ 


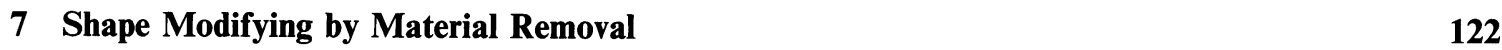

$\begin{array}{lll}7.1 & \text { Introduction } & 122\end{array}$

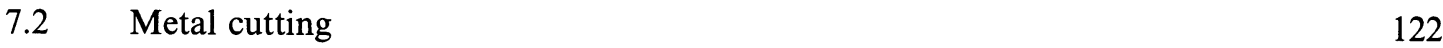

$\begin{array}{ll}\text { 7.2.1 Cutting mechanism } & 123\end{array}$

$\begin{array}{lll}\text { 7.2.2 Chip formation } & 123\end{array}$

$\begin{array}{lll}7.2 .3 & \text { Tool geometry } & 124\end{array}$

$\begin{array}{lll}7.2 .4 & \text { Tool materials } & 125 \\ 7.2 .5 & \text { Cutting fluids } & 126\end{array}$

$\begin{array}{lll}7.2 .5 & \text { Cutting fluids } & 126\end{array}$

$\begin{array}{llr}7.2 .6 & \text { Tool wear } & 127\end{array}$

$\begin{array}{llr}\text { 7.2.7 Tool life } & 128\end{array}$

$\begin{array}{lll}7.2 .8 & \text { Cutting economics } & 128\end{array}$

$\begin{array}{lll}7.2 .9 & \text { Cutting forces } & 129\end{array}$

7.2.10 Machine tools $\quad 132$

$\begin{array}{lll}7.3 & \text { Abrasive machining } & 136\end{array}$

$\begin{array}{lll}\text { 7.3.1 Grinding } & 136\end{array}$

$\begin{array}{lll}\text { 7.3.2 Honing } & 137\end{array}$

$\begin{array}{lll}\text { 7.3.3 Superfinishing } & 137\end{array}$

$\begin{array}{lll}7.3 .4 & \text { Lapping } & 137\end{array}$

$\begin{array}{lll}7.4 & \text { Other methods } & 137\end{array}$

$\begin{array}{ll}\text { 7.4.1 Electrochemical machining } & 137\end{array}$

$\begin{array}{ll}\text { 7.4.2 Electric discharge machining } & 137\end{array}$

$\begin{array}{ll}\text { 7.4.3 Laser profiling } & 138\end{array}$

$\begin{array}{lll}7.5 & \text { Summary } & 138\end{array}$

$\begin{array}{lll}7.6 & \text { Questions } & 138\end{array}$

8 Shape Modifying: Retaining Material $\quad 139$

$\begin{array}{lll}8.1 \quad \text { Introduction } & 139\end{array}$

$\begin{array}{lll}8.2 & \text { Casting } & 139\end{array}$

$\begin{array}{lll}\text { 8.2.1 Terminology } & 140\end{array}$

8.2.2 Sand casting 141

$\begin{array}{lll}\text { 8.2.3 Shell moulding } & 142\end{array}$

$\begin{array}{lll}\text { 8.2.4 Investment casting } & 143\end{array}$

$\begin{array}{lll}\text { 8.2.5 Die casting } & 143\end{array}$

$\begin{array}{lll}8.3 & \text { Powder forming } & 145\end{array}$

$\begin{array}{lll}\text { 8.3.1 Powder manufacture } & 145\end{array}$

$\begin{array}{lll}\text { 8.4.2 Compaction } & 145\end{array}$

$\begin{array}{lll}\text { 8.4.3 Sintering } & 146\end{array}$

8.3.4 Hot isostatic pressing $\quad 146$

$\begin{array}{lll}8.4 & \text { Plastic deformation } & 146\end{array}$

8.4.1 Hot and cold working 146

$\begin{array}{lll}8.4 .2 & \text { Forging } & 146\end{array}$

$\begin{array}{lll}\text { 8.4.3 Rolling } & 147\end{array}$

$\begin{array}{lll}\text { 8.4.4 Extrusion } & 148\end{array}$

8.4.5 Sheet metal processes 148

8.5 Processes for non-metals $\quad 151$

8.5.1 Plastics 151

$\begin{array}{lll}\text { 8.5.2 Ceramics } & 153\end{array}$

$\begin{array}{lll}\text { 8.5.3 Composites } & 154\end{array}$ 
$\begin{array}{lll}\text { 8.6 Summary } & 154\end{array}$

$\begin{array}{lll}8.7 & \text { Questions } & 155\end{array}$

9 Joining Techniques $\quad 156$

$\begin{array}{lll}9.1 & \text { Introduction } & 156\end{array}$

$\begin{array}{lll}9.2 & \text { Non-permanent joints } & 157\end{array}$

$\begin{array}{lll}9.3 & \text { Semi-permanent joints } & 157\end{array}$

$\begin{array}{lll}\text { 9.3.1 Mechanical } & 157\end{array}$

9.3.2 Liquid-solid bonding (brazing and soldering) 157

$\begin{array}{lll}9.4 & \text { Permanent joints } & 159\end{array}$

$\begin{array}{lll}\text { 9.4.1 Welding } & 159\end{array}$

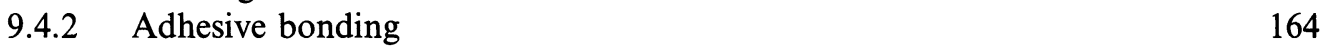

$\begin{array}{lll}9.5 & \text { Summary } & 165\end{array}$

$\begin{array}{lll}9.6 & \text { Questions } & 167\end{array}$

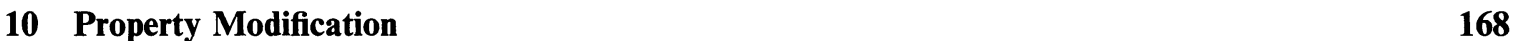

$\begin{array}{lll}10.1 & \text { Introduction } & 168\end{array}$

$\begin{array}{llr}10.2 & \text { Heat treatment } & 169\end{array}$

$\begin{array}{lll}\text { 10.2.1 Structure of steel } & 169\end{array}$

$\begin{array}{lll}10.2 .2 & \text { Stress relieving } & 170\end{array}$

$\begin{array}{lll}10.2 .3 & \text { Annealing } & 170\end{array}$

$\begin{array}{lll}10.2 .4 & \text { Normalising } & 170\end{array}$

10.2.5 Hardening 171

10.2.6 Tempering 171

$\begin{array}{lll}10.2 .7 & \text { Surface hardening } & 171\end{array}$

$\begin{array}{lll}\text { 10.2.8 Case hardening } & 171\end{array}$

$\begin{array}{lll}10.3 & \text { Surface finishing } & 171\end{array}$

10.3.1 Mechanical 171

$\begin{array}{ll}\text { 10.3.2 Coating } & 172\end{array}$

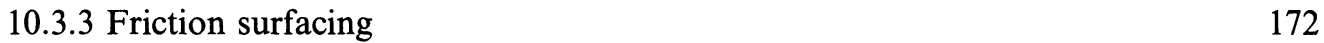

$\begin{array}{lll}10.4 & \text { Summary } & 173\end{array}$

$\begin{array}{lll}10.5 & \text { Questions } & 173\end{array}$

11 Quality Control $r$

$\begin{array}{lll}11.1 & \text { Introduction } & 174\end{array}$

$\begin{array}{ll}11.2 & \text { Inspecting geometric features } \\ & 11.2 .174\end{array}$

$\begin{array}{lll}\text { 11.2.1 Inspection equipment } & 174\end{array}$

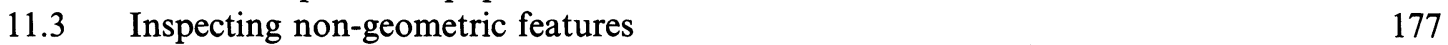

$\begin{array}{lll}\text { 11.3.1 Proof testing } & 177\end{array}$

$\begin{array}{lll}\text { 11.3.2 Visual inspection } & 177\end{array}$

$\begin{array}{lll}\text { 11.3.3 Dye penetrant } & 177\end{array}$

$\begin{array}{lll}\text { 11.3.4 Magnetic particle } & 178\end{array}$

$\begin{array}{lll}\text { 11.3.5 } & \text { Radiographic } & 178\end{array}$

$\begin{array}{lll}\text { 11.3.6 Ultrasonic, acoustic } & 178\end{array}$

$\begin{array}{lll}\text { 11.3.7 Leak detection } & 178\end{array}$

11.4 Traceability 178

11.5 Process control and feedback $\quad 179$

$\begin{array}{lll}\text { 11.5.1 Process capability } & 179\end{array}$ 


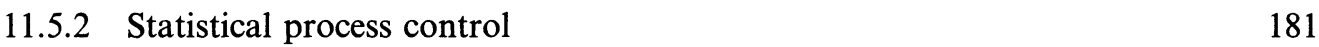

$\begin{array}{ll}\text { 11.5.3 Quality control charts } & 182\end{array}$

$\begin{array}{lll}11.6 & \text { Summary } & 183\end{array}$

11.7 Questions 183

\section{Part III Effective Integration of Design and Manufacture}

12 Designing for Manufacturing Processes and Materials 187

$\begin{array}{lll}12.1 & \text { Introduction } & 187\end{array}$

$\begin{array}{lll}12.2 & \text { Casting } & 187\end{array}$

$\begin{array}{ll}\text { 12.2.1 When to use castings } & 187\end{array}$

$\begin{array}{ll}\text { 12.2.2 Which casting process? } & 187\end{array}$

$\begin{array}{ll}12.2 .3 \text { General design points } & 189\end{array}$

$\begin{array}{lll}12.3 & \text { Forging } & 190\end{array}$

12.3.1 When to use forgings 190

$\begin{array}{ll}\text { 12.3.2 Which forging process? } & 191\end{array}$

12.3.3 General design points 191

$\begin{array}{lll}12.4 & \text { Powder metallurgy } & 192\end{array}$

12.4.1 When to use powder metallurgy 192

12.4.2 General design points 192

$\begin{array}{lll}12.5 & \text { Material removal } & 193\end{array}$

12.5.1 When to machine 193

$\begin{array}{ll}\text { 12.5.2 Which machining process? } & 194\end{array}$

12.5.3 General design points 195

$\begin{array}{lll}12.6 & \text { Plastics } & 197\end{array}$

$\begin{array}{ll}\text { 12.6.1 When to use plastics } & 197\end{array}$

12.6.2 Which plastic? 197

12.6.3 General design points 197

$\begin{array}{lll}12.7 & \text { Ceramics } & 199\end{array}$

$\begin{array}{lll}12.8 & \text { Composites } & 199\end{array}$

12.8.1 When to use composites 199

$\begin{array}{ll}12.8 .2 \text { General design points } & 200\end{array}$

$\begin{array}{lll}12.9 & \text { Summary } & 201\end{array}$

$\begin{array}{ll}12.10 & \text { Questions } \\ & 201\end{array}$

13 Designing for Joining and Assembly 202

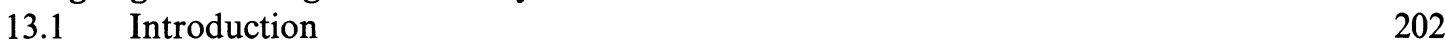

13.2 Choice of process 203

13.3 Heat application 203

13.3.1 Welding 203

$\begin{array}{ll}\text { 13.3.2 Brazing and soldering } & 207\end{array}$

$\begin{array}{lll}13.4 & \text { Chemical application } & 207\end{array}$

$\begin{array}{ll}\text { 13.4.1 Adhesives } & 207\end{array}$

13.5 Mechanical joints 208

13.5.1 Integral features 208

$\begin{array}{ll}\text { 13.5.2 Threaded fasteners } & 212\end{array}$

13.5.3 Non-threaded fasteners 216 
$\begin{array}{lll}13.6 & \text { Automated assembly } & 216\end{array}$

$\begin{array}{lll}13.7 & \text { Summary } & 218\end{array}$

$\begin{array}{lll}13.8 & \text { Questions } & 219\end{array}$

14 Influences on Design and Manufacturing Choices $\quad 220$

$\begin{array}{lll}14.1 & \text { Introduction } & 220\end{array}$

$\begin{array}{lll}14.2 & \text { Aesthetics } & 220\end{array}$

\begin{tabular}{lll}
14.3 & Ergonomics & 222 \\
\hline
\end{tabular}

14.4 Quantity 223

$\begin{array}{lll}14.5 & \text { Safety } & 225\end{array}$

$\begin{array}{ll}14.6 & \text { Strength, fatigue }\end{array}$

$\begin{array}{llr}14.7 & \text { Corrosion } & 229\end{array}$

$\begin{array}{lll}14.8 & \text { Environment } & 230\end{array}$

14.9 Conflict, compromise $\quad 236$

$\begin{array}{lll}14.10 & \text { Summary } & 237\end{array}$

15 Analysis of Existing Designs and Manufacturing Processes $\quad 238$

$\begin{array}{lll}15.1 & \text { Introduction } & 238\end{array}$

$\begin{array}{lll}15.2 & \text { Value analysis } & 238\end{array}$

$\begin{array}{lll}15.3 & \text { Summary } & 247\end{array}$

$\begin{array}{lll}15.4 & \text { Questions } & 248\end{array}$

16 Systems for Controlling Design and Manufacture $\quad 249$

$\begin{array}{lll}16.1 & \text { Introduction } & 249\end{array}$

$\begin{array}{lll}16.2 & \text { Primary tasks } & 249\end{array}$

$\begin{array}{ll}\text { 16.2.1 Company aims, objectives and strategy } & 250\end{array}$

$\begin{array}{lll}\text { 16.2.2 } & \text { Future products and processes } & 250\end{array}$

16.2.3 Develop and implement new products and processes $\quad 251$

$\begin{array}{ll}\text { 16.2.4 Obtain customer orders } & 251\end{array}$

16.2.5 Manufacture products and despatch to customers $\quad 252$

16.2.6 Recover payment from the customer $\quad 254$

$\begin{array}{lll}\text { 16.2.7 Service the system } & 254\end{array}$

$\begin{array}{lll}16.3 & \text { Information flows } & 256\end{array}$

16.3.1 Types of order $\quad 256$

$\begin{array}{ll}\text { 16.3.2 Special order } & 256\end{array}$

$\begin{array}{ll}\text { 16.3.3 Standard product order } & 259\end{array}$

$\begin{array}{lll}16.4 & \text { Control systems } & 261\end{array}$

16.4.1 Engineering changes 261

$\begin{array}{ll}\text { 16.4.2 Efficiency measurement } & 262\end{array}$

$\begin{array}{lll}\text { 16.4.3 Stock control } & 263\end{array}$

$\begin{array}{ll}\text { 16.4.4 Material stock movements } & 264\end{array}$

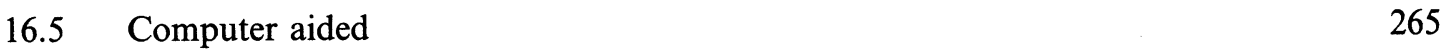

$\begin{array}{lll}\text { 16.5.1 Background } & 265\end{array}$

$\begin{array}{ll}\text { 16.5.2 Product development } & 266\end{array}$

$\begin{array}{ll}\text { 16.5.3 Manufacturing control } & 270\end{array}$

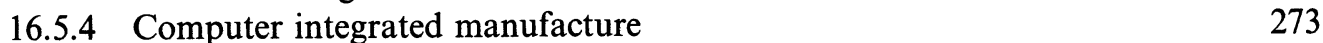

$\begin{array}{lll}16.6 & \text { Summary } & 273\end{array}$

$\begin{array}{lll}16.7 & \text { Questions } & 274\end{array}$ 
17 The Business Context (Organisation and Costing) 275

$\begin{array}{lll}17.1 & \text { Introduction } & 275\end{array}$

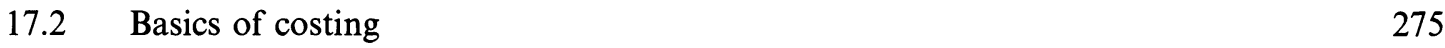

$\begin{array}{lll}17.2 .1 & \text { Profit } & 275\end{array}$

$\begin{array}{lll}17.2 .2 & \text { Income } & 275\end{array}$

$\begin{array}{lll}17.2 .3 & \text { Outgoings } & 276\end{array}$

$\begin{array}{lll}\text { 17.2.4 Fixed and variable costs } & 276\end{array}$

$\begin{array}{lll}17.3 & \text { Applications } & 277\end{array}$

$\begin{array}{lll}\text { 17.3.1 Changes to an existing product } & 277\end{array}$

17.3.2 Introducing a new process or piece of plant 281

$\begin{array}{lll}\text { 17.3.3 Introducing a new product } & 284\end{array}$

$\begin{array}{lll}17.4 & \text { Quantity } & 287\end{array}$

$\begin{array}{lll}17.5 & \text { Summary } & 288\end{array}$

$\begin{array}{lll}17.6 & \text { Questions } & 288\end{array}$

\section{Part IV Appendices}

A Units and Definitions $\quad 293$

$\begin{array}{lll}\text { A.1 Introduction } & 293\end{array}$

$\begin{array}{lll}\text { A.2 Units } & 293\end{array}$

$\begin{array}{lll}\text { A.2.1 SI system } & 293\end{array}$

A.2.2 Imperial system 295

$\begin{array}{lll}\text { A.2.3 Unity brackets } & 298\end{array}$

A.2.4 Dimensional analysis $\quad 298$

$\begin{array}{ll}\text { A.3 Equations of motion } & 299\end{array}$

$\begin{array}{lll}\text { A.4 Force, motion and work } & 300\end{array}$

A.4.1 Newton's laws of motion $\quad 300$

$\begin{array}{ll}\text { A.4.2 Work, energy and power } & 300\end{array}$

B Data Tables $\quad 301$

B.1 Surface roughness $\quad 302$

B.2 Relative costs of processes $\quad 303$

$\begin{array}{lll}\text { B.3 Selected ISO fits: hole basis } & 304\end{array}$

$\begin{array}{ll}\text { B.4 Selected ISO fits: shaft basis } & 305\end{array}$

B.5 Properties of sections $\quad 306$

$\begin{array}{lll}\text { B.6 Deflections and moments for simple beams } & 308\end{array}$

$\begin{array}{lll}\text { B.7 } & \text { Friction coefficients } & 309\end{array}$

$\begin{array}{ll}\text { B.8 Corrosion: electrochemical series } & 310\end{array}$

B.9 Materials data (steels, non-ferrous metals, non-metals) 311

$\begin{array}{lr}\text { C Costing Calculations } & 314\end{array}$

$\begin{array}{lr}\text { D Solutions } & 316\end{array}$

$\begin{array}{lr}\text { E Equivalent Standards } & 320\end{array}$

Bibliography and Further Reading

$\begin{array}{ll}\text { Index } & 322\end{array}$ 


\section{Preface}

In the early 1980s I worked for an engineering company, designing and manufacturing products. The design office contained the 'experts' in design, who created drawings for new products. When complete, these were passed to production engineering, located in a separate building remote from the design offices, where the 'experts' in production resided. They then modified the designs, so that they could be more easily manufactured. Much time was lost through the many conflicts over details that were 'vital' to the functioning of the final product and at the same time 'impossible' to manufacture. The solution was to combine the design and production engineering departments and locate them together, adjacent to the manufacturing plant. The design and production 'experts' were both involved in the product development process, working together as a team, rather than blaming each other for shortcomings in the products. The end result for the company was that new products could be introduced more quickly. In addition, they generally had fewer teething problems and tended to be more profitable. The moral is obvious.

The teaching of engineering tends to be very compartmentalised, with different aspects being taught by experts in various fields. Although there are many good reasons for this, I believe that we should never lose sight of the fact that in the real world, engineering problems rarely fit precisely into the compartments of knowledge as taught. It is therefore vital that the importance of integration across the subject boundaries is covered from an early stage in the teaching of engineering.

This book has been written for engineering students, recognising the need both to introduce basics skills and techniques and to show how they would be used in an integrated fashion.

Chapter 1 introduces the route by which an idea is converted into a product, using a case study to highlight the need for an understanding of the process. This is followed by the three main parts of the text.

The first part concentrates on the basics of design. The content includes the engineering drawing, tolerances, a range of standard components, the product design specification, and methods of generating and evaluating ideas. The second part concentrates on basic manufacturing techniques. These include material removal processes, casting, deforming, powder metallurgy, processes for metals and non-metals, joining techniques, processes for altering material properties, and an introduction to the concepts of quality.

The third part builds upon the basics, integrating aspects from the first two parts. Chapter 12, for example, looks at why a particular manufacturing process might be selected, and how the designer might take full advantage of the chosen process. Chapter 13 covers the application of joining techniques, discussing topics such as when and how should an interference joint be selected and designed, and how the use of automated assembly processes can influence the design. The next chapter discusses, largely through case studies, how a variety of influences result in engineering compromises between design and manufacture. Chapter 15 shows, again through the use of case studies, how value analysis can be used to improve both the component design and the manufacturing process. Chapter 16 takes an overall view of an organisation designing and manufacturing products, examining the systems used, how control is maintained, and how computers can be used to help. The final chapter examines the business context, costing systems, and how decisions might be made when selecting a new piece of manufacturing plant, or introducing a new product.

Recognising that units are a common source of error for students, Appendix A includes defini- 
tions, conversion factors, and sections on unity brackets and dimensional analysis.

Revision questions are included, where appropriate, at the end of each chapter. Solutions can be found either in the text, as directed, or in appendix D. Throughout there are various references to British Standards. Equivalent, or similar, International Standards are listed in appendix E.

The book builds on much that I have learned working as an engineer over the past two decades, but has only been possible because of the help, co-operation, suggestions and tolerance from a variety of colleagues, too many to mention individually. I would, however, like to thank, in particular, John Bishop for his general help and advice, and specifically for his contribution, with the assistance of $3 \mathrm{M}$, to the section on adhesives. I would also like to single out Bill Puttick for his helpful comments and advice on the chapter on metal cutting. Many thanks, Bill. Last, but not least, I must also thank my family, who have shown remarkable tolerance whilst I concentrated on 'the book', ignoring them for far too long.

ROD BLACK 


\section{Acknowledgements}

The author and publishers would like to thank the following for the use of copyright material:

Figures 4.17, 4.18, 4.19, 4.20, 4.21 reprinted with the permission of SEED (Sharing Experience in Engineering Design) and McGraw-Hill Book Company Europe, from their publication, $\mathrm{K}$. Hurst, Rotary Power Transmission Design, 1994.

Figure 5.5, Table 5.2 reproduced with the permission of the Institution of Electrical Engineers from $A$ Guide to Design for Production, published by The Institution of Production Engineers, 1984.
Figure 4.38 reprinted with the permission of SKF (UK) from their General Catalogue 1989.

Figures 3.21, 3.22, B.2 and Tables B.3, B.4 are extracts from British Standards and are reproduced with the permission of BSI. Complete copies can be obtained by post from BSI Sales, Linford Wood, Milton Keynes MK14 6LE. 